ORIGINAL ARTICLE

PRACA ORYGINALNA

\title{
THE KEY ROLE OF PANCREATIC FIBROSIS SEVERITY IN THE SURGICAL TREATMENT ALGORITHM OF PATIENTS WITH CHRONIC PANCREATITIS
}

DOI: 10.36740/WLek202002105

\author{
Oleh E. Kanikovskiy, Ihor V. Pavlyk, Iryna V. Oliinyk, Vasyl V. Mosondz \\ NATIONAL PIROGOV MEMORIAL MEDICAL UNIVERSITY, VINNYTSIA, UKRAINE
}

\begin{abstract}
The aim of the work was to improve the results of surgical treatment of complicated forms of chronic pancreatitis.

Materials and methods: The results of surgical treatment of 181 patients with complicated forms of chronic pancreatitis have been analyzed. All these patients were treated in surgical clinic of 2 nd medical faculty of National Pyrogov Memorial Medical University in Vinnytsya.

Results: It is possible preoperative indirectly assess the severity of fibro- degenerative changes in pancreas, that includes 1 - the definition of pain type; 2 - CT or MRI (MarseillesRome classification); 3 - assessment of the stage of chronic pancreatitis (Büchler classification); 4 - patient\&\#39; 5 nutrition status and preoperative differential diagnosis with pancreatic cancer; 5 - assessment of the fibrosis severity (elastomers). The key point in treatment depended on intraoperative examination: detection of strictures of the main pancreatic duct (pacemaker of chronic pancreatitis); the tissue pressure resistance to the liquid, which is the maximum value\&gt; $200 \mathrm{mmHg}$, in the region of stricture and falls in other parts of the pancreas; pressure in the main pancreas duct, which rises only in $59.5 \%$ of patients. The head of the pancreas was involved in the pathological process in $83.8 \%$, in $16.2 \%$ it was isolated in the isthmus or the body and tail of the pancreas. In general, the distal pancreas was involved in 37.8\%. At computer morphometry of histological samples, the area of connective tissue fields reached $81.4 \pm 6.62 \%$, preserved exocrine part in $4.87 \pm 1.62 \%$, endocrine $-1.92 \pm 0.12 \%$, total area of ducts $-6,47 \pm 1.12 \%$. Conclusions: The combined Frey-Izbickiy local resection provides a wide excision of the pacemaker (stricture) of chronic pancreatitis. In case of extrapancreatic complications or repeated surgical interventions on the pancreas due to chronic pancreatitis, this effect can be achieved by pancreatoduodenal resection.
\end{abstract}

KEY WORDS: chronic pancreatitis, tissue resistant pressure

Wiad Lek. 2020;73(2):235-238

\section{INTRODUCTION}

The main morphological features of chronic pancreatitis are fibrosis and atrophy of the pancreas. The main clinical manifestation of chronic pancreatitis is pain, exo- and endocrine insufficiency of the pancreas. During the last century, the experience of performing various operations in chronic pancreatitis was accumulated, from the simple Partington-Roshe drainage operation, various modifications of local resections based on the Frey operation, Beger's duodenum-saving resection of the head of the pancreas to well-known pancreatoduodenal resection. Despite the significant progress in understanding of the approaches to the surgical treatment of chronic pancreatitis, currently no clear demarcation between the choices of one or another procedure [1-4].

\section{THE AIM}

The aim - to define the stage of pancreatic fibrosis as key role factor to choose the type of surgical treatment of patient with chronic pancreatitis.

\section{MATERIALS AND METHODS}

The results of surgical treatment of 181 patients with chronic pancreatitis complicated with pancreatic hypertension from
2000 to 2018 submitted. Mean age were $46,0 \pm 13,7$ year, male - 138, female -41 . According to TIGAR-O classification in $162(90 \%)$ patients chronic pancreatitis occur after attack of acute pancreatitis, and 172 (95\%) patients were alcohol abused. According to Marcel-Rome classification: calcification chronic pancreatitis had 21 patients, obstructive -34 , inflammation form-2, fibrosis-12, cyst -112 .

The stage of $\mathrm{CP}$ was determined according to the Büchler classification (2009): "B" stage were in $22.65 \%$ (41 patients), "C" stage - 77.35\% (140). Among patients with C stage pancreatogenic diabetes was noted in 38 patients.

In 37 patients during surgical procedure, we check a tissue resistant pressure with Stryker tissue pressure monitor and pressure inside the main pancreatic duct. Histologically fibrosis define into 4 stages according to Stolt.

All patients had surgical correction. Different types of pancreatic resection have done in 69 patients. In other 112 patients: internal drainage of the cyst (61), laparoscopic cystogastrostomy (5), combine internal-external drainage (18) and external drainage (29).

Statistical processing of the material was carried out using Statistica (Version 6.0 StatSoft Inc., USA). The reliability of the data difference was determined using the student's $\mathrm{t}$-criterion. The results were considered statistically significant at $\mathrm{p}<0.05$. 


\section{RESULTS}

The course of chronic pancreatitis is continuously recurrent, and with each new attack of the disease, the degree of morphological changes in the pancreas is deepened. This concept makes it possible to introduce into the clinical practice the algorithm for the examination and treatment of such patients, which is built on the preoperative and intraoperative stages of the severity of pathomorphological changes, as well as postoperative histological confirmation of these indicators.

The preoperative stage includes 1 - the definition of the type of pain; 2 - CT with evaluation of fibrosis-degenerative changes and complications according to the Marcel-Rome classification; 3 - evaluation of the stage of chronic pancreatitis according to the Büchler classification (serum glucose level, feces elastase level); 4 - patient's nutritional state (BMI); level of CA 19-9 and CA 50; 5 - preoperation evaluation of the degree of fibrosis (FibroScan).

Numerous studies have shown [2.3] that it is possible to indirectly evaluate the severity of fibro-degenerative changes of the pancreas according to the type of pain prior to the surgical stage. Important several principles of pain gradation: 1 - permanent or episodic; 2 - is purchased medically or not. Rudolf M. Ammann et al., 1999, divided the pain syndrome into category A - episodic severe pain with the existing painless periods; $\mathrm{B}$ - constant with episodes of severe pain, requiring hospitalization.

Dependence of pain type to CP severity were checked in 62 patients. All patients with calcification CP (5), multiple strictures of main pancreatic duct (7), large (12) and infected (5) pancreatic cyst had pain type B on the time of admission to the hospital. Patients with pancreatic head alone stricture (4), small and medium (26) pancreatic cyst had type A pain. One patient with fibrosis of the pancreas had type $B$ and one - type A pain. And 1 patient more with pancreatic head alone stricture had type B pain.

It is important to evaluate the so-called pacemaker of chronic pancreatitis - strictures of main pancreatic duct. Among 37 patients, isolated strictures were noted in 13 (35.1\%) and multiple in 24 (64.9\%) patients. The head of the pancreas was involved in the pathological process in $83.8 \%$ (31 patients), that is, the head of the pancreas was not always a pacemaker of chronic pancreatitis, in $16.2 \%$ (6 patients) it was isolated in the isthmus (4) or the body and tail ( 2) of the pancreas. In general, distal part of the pancreas were injured in $37.8 \%$.

Nutritive status of patients differed according to the form of chronic pancreatitis. In patients with calcifying form - BMI were $17.2 \pm 1.6$; Obstructive - 18,1 $\pm 2,1$; inflammatory $-24,7 \pm 1,8$; fibrosis $23.8 \pm 1.2$; cysts $-25.3 \pm 1.5$.

In the preoperative period In 20 patients, were determined the density of the panreas with ultrasound elastography (Fibroscan). The data of elastography clearly depended on the form of CP. So in 6 patients with CP with multiple strictures (4) and calculous (2), the density of the pancreas were $25,6 \pm 2,3 \mathrm{kPa}$. All of these patients had type $B$ of the pain syndrome, as well as the intraoperative tissue resistant pressure $>200 \mathrm{~mm} \mathrm{Hg}$ in all sections of the pancreas and histologically confirmed type 4 fibrosis.
In 2 patients with chronic pancreatitis with isolated stricture of the pancreatic duct, the elastography rates were $23.7 \pm 1.5 \mathrm{kPa}$. In these patients, the type $\mathrm{B}$ of the pain syndrome was noted, the TRP consisted of $200 \mathrm{~mm}$ $\mathrm{Hg}$ in the head of the PO, in the body and tail TRP were > $120 \mathrm{~mm}$ Hg. Histologically, in the head type 4, in the body and the tail 3 types of fibrosis was noted.

In 2 patients with chronic pancreatitis with type $B$ pain and with increasing size of the pancreas without complications and dilatation of the main pancreatic duct, the density of the pancreas were $21 \pm 0.5 \mathrm{kPa}$. These patients were not operated. However, these data make possible to draw a clear line in choosing a method for treating patients with CP between gastroenterologists and surgeons. Thus, patients with type $\mathrm{B}$ pain and density values more than $20 \mathrm{kPa}$ require surgical treatment.

The other 10 patients were a control group. So, for 2 volunteers, norm parameters for elastoscopy of software were determined, which is $4-6 \mathrm{kPa}$, in 8 patients who showed manifestations of HP in the background of housing and communal services $-16,7 \pm 1,7 \mathrm{kPa}$. 11 of these patients were marked with type A pain syndrome.

In patients with calcifying forms of $\mathrm{CP}$ (16 patients), total calculous was noted in $87.5 \%$ (14), the isolated stricture of the head was noted in $37.5 \%$ (6). The isolated stricture of other departments (tail of the pancreas) was noted only in 2 (5.4\%) patients. It should be noted that in all cases, the calculus zone was combined with the multiple strictures of this part of the pancreas.

One of the most effective intraoperative method to detect the severity of pancreatic fibrosis are measurement of tissue resistant pressure. In all cases tissue resistant pressure was highest it the place of main pancreatic duct stricture $(>200$ $\mathrm{mm} . \mathrm{Hg}$ ), as in other parts of the pancreas it decrease till $120 \mathrm{~mm} . \mathrm{Hg}$ and less. Ductal pressure was increased only in $22(59,5 \%)$ patients. All patients histologically had 4 grade of fibrosis in the stricture place. tissue resistant pressure was $>200 \mathrm{~mm}$.Hg in the head of the pancreas only in 30 $(81,1 \%)$ patients. In 7 patients location of the stricture were in the pancreatic isthmus and tissue resistant pressure in that place proved zone of severe fibrosis. Patient with calculosis (head -6 , total-8) and obstructive chronic pancreatitis (multiple stricture of main pancreatic duct-8) had tissue resistant pressure as high as $>200 \mathrm{~mm}$.Hg in all part of the pancreas indicating the total pancreatic fibrosis.

In all patients undergoing surgical intervention, in the place of stricture of main pancreatic duct were determined 4 degree of pancreatic fibrosis - total fibrosis, which was described as a significant fibrosis degradation of the pancreas with complete destruction of exocrine parenchyma. At computer morphometry of histological samples, the area of connective tissue fields reached $81.4 \pm 6.62 \%$, preserved pancreatic exocrine fraction $-4.87 \pm 1.62 \%$, endocrine $1.92 \pm 0.12 \%$, total area of ducts $-6,47 \pm 1.12 \%$.

\section{DISCUSSION}

The results of the research indicate that pancreatic hypertension syndrome is not so much an isolated increase 


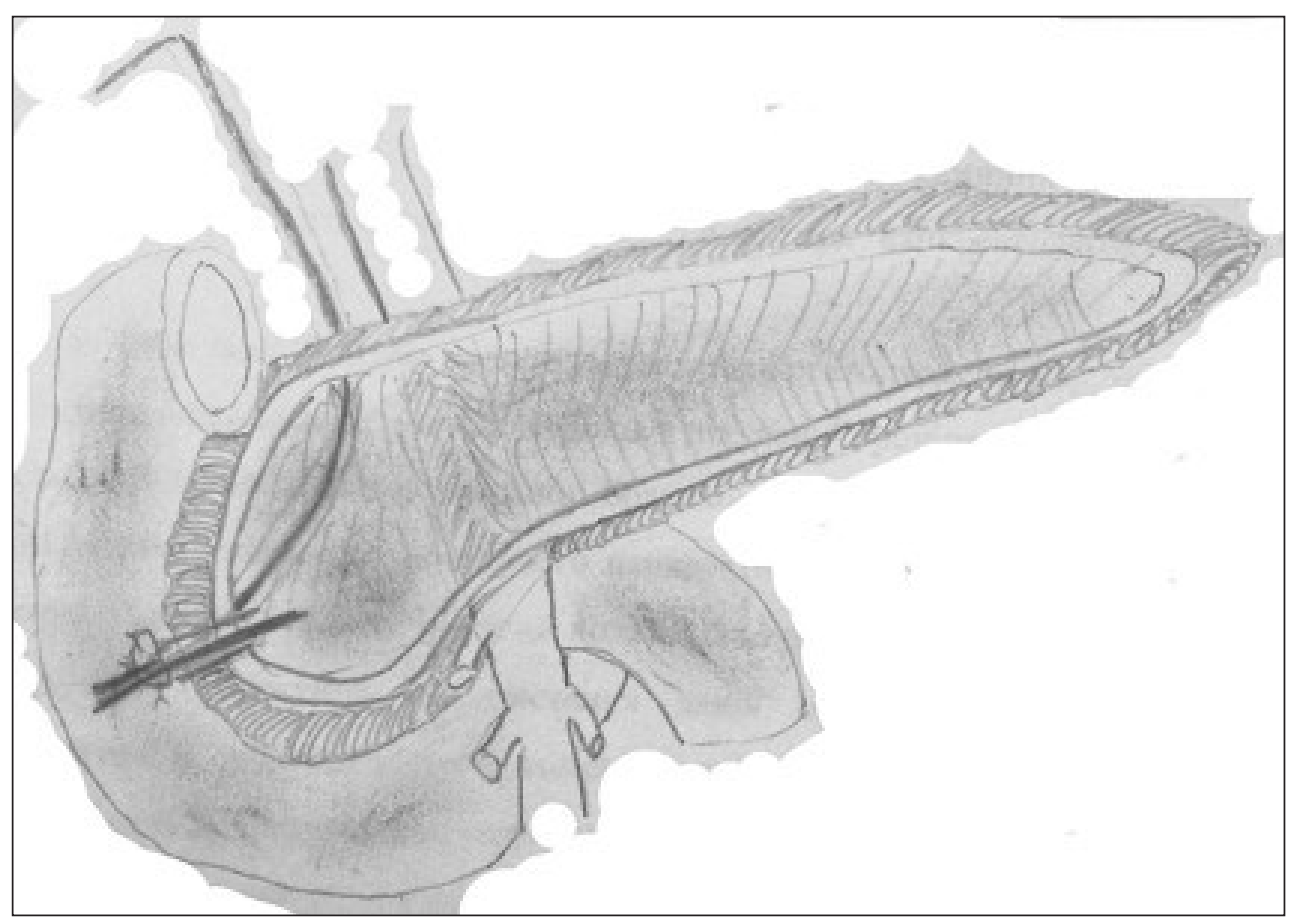

Figure 1. Scheme of Frey-Izbitsky operation with transpancreatic papilloprotectomy. of pressure inside the main pancreatic duct as the severe fibrotic process in the tissue of the pancreas. Therefore, in surgical correction of pancreatic hypertension syndrome, it is important not only to eliminate intraductal pressure, but also to maximize the opening of secondary and tertiary pancreatic ducts and to remove the pacemaker $\mathrm{CP}$ by cutting out the stricture of main pancreatic duct. Moreover, the excision of parenchyma indicated in zone of maximal tissue resistance pressure $(>200 \mathrm{~mm} \mathrm{Hg})$, but in a place where this pressure $<100 \mathrm{~mm} \mathrm{Hg}$ it is enough to do simple drainage operation [5-6].

For the head of the pancreas, it is important to unite of the main pancreatic duct and the duct of the uncinate process into one cavity $[7,8,9]$. Thus, a local resection of the pancreas meets all tasks sated above the surgical treatment. The proposed operation technique combines the idea of two operations of Frey and Izbicki and expands them due to the wide excision of parenchyma with strictures as well as the excision of the main pancreatic duct for the opening of the secondary and tertiary ducts. (Patent of Ukraine 103273, September 25, 2013).

Technically, this operation perform as follows: first, we do $\mathrm{U}$-shaped excision of the walls of the main pancreatic duct with strictures, with the edges being $5 \mathrm{~mm}$ above the upper and lower edges of the body and the tail of the pancreas. The depth of the exsision is the back wall of the main pancreatic duct. The next step is the excavation of the pancreatic head. The cutting edge is about $5 \mathrm{~mm}$ in the upper, lateral and lower edges of the pancreatic head. The depth of the excision is the posterior wall of the main pancreatic Duct and the duct of the uncinated process, and the common bile duct. Intraparenchymal part of the common bile duct is released and released from surrounding strictures. The common bile duct is not disclosed, which allows you to not perform biliodigestive anastomosis. Thus, at the end of the excision in the area of the pancreatic head keep a thin, $5 \mathrm{~mm}$ thickness, rim of parenchyma. (Fig. 1). We recommend this operation supplement with transpanreatic papilloprotectomy, to restore the physiological passage of pancreatic juice to the duodenum (patent of Ukraine No. 115291, 10.10.2017). The Frey-Izbicki technique were done in 52 patients with chronic pancreatitis. Postoperative mortality were $0 \%$, morbidity $-13,46 \%$ (7 patients). Bleeding from zone of pancreatojejuno anastomosis occur in 2 patients due to were done 1 relaparotomy; pancreatic fistula (grade B) in 2 patients - all were treated conservatively; abdominal abscess in 1 patient were treated with puncture and drainage under ultrasound control; abdominal wound infection in 2 patients were treated with the drainage of postoperative wound.

The surgical treatment algorithm depend on intraoperative tissue resistant pressure and ductal pressure. The Frey (6) procedure we used if tissue resistant pressure $>200 \mathrm{~mm} . \mathrm{Hg}$ in the pancreatic head, in other parts $<120$ $\mathrm{mm} . \mathrm{Hg}$., ductal pressure - high or normal. The Izbicki (2) procedure -if main pancreatic duct were obliterated with tissue resistant pressure $>200 \mathrm{~mm}$.Hg in all parts of the pancreas. The Frey-Izbicki (52) procedure- in case of multiple stricture of main pancreatic duct, total calculosis, tissue resistant pressure $>200 \mathrm{~mm}$. Hg in all parts of the pancreas, ductal pressure high. Pancreatoduodenal resection (PDR) (9) done in case of high risk of pancreatic cancer, extrapancreatic complication of fibrotic degenerative chronic pancreatitis, recurrent surgical procedures on pancreas. Corporocaudal pancreatic resection (CCR) (2) done in case of high risk of pancreatic malignancy or severe fibrotic degenerative process of the distal part of the pancreas.

Postoperative mortality was $0 \%$. Postoperative morbidity occur in $18,85 \%$ (13 patients) for which 3 relaparotomies 
were performed. Among the complications, abdominal bleeding occur in $2.9 \%$ ( 2 patients), pancreatic fistula in $7.25 \%$ (5), abdominal abscesses in 4.3\% (3), and postoperative wound infection in $4.3 \%$ (3).

The analysis of long-term results carried out in $24 \mathrm{pa}-$ tients for 6-36 months. We did not report the recurrence of pancreatic hypertension after using the Frey-Izbicki technique. According to the indicators of the SF-36 questionnaire in operated patients in 6 months or more, the "pain intensity" $(\mathrm{P})$ was $3.2 \pm 0.19$, which was lower compared to preoperative parameters $(5.3 \pm 0.5 ; \mathrm{p}<0.05)$.

\section{CONCLUSIONS}

The pacemaker of CP should considered as zone of maximal fibrosis that not always located in the pancreatic head. Therefore, surgical procedure should not only decreas ductal pressure and make local resection of the head of the pancreas but wide excision of the pancreatic stricture with the opening of the secondary and tertiary ducts (Frey-Izbicki procedure). Moreover, excision should perfomed in zone of maximal TRP (>200 mm.Hg), but in place TRP lower $120 \mathrm{~mm} . \mathrm{Hg}$ it is enough to do simple draining procedure.

\section{REFERENCES}

1. Jorge D Machicado, Vinciane Rebours, Dhiraj Yadav. Epidemiology of Chronic Pancreatitis. Pancreapedia: Exocrine Pancreas Knowledge Base. 2016 July; 28. Mode of access: https://www. pancreapedia.org/reviews/epidemiology-of-chronic-pancreatitis (D0l: 10.3998/panc.2016.13).

2. Anderson M.A., Akshintala V., Albers K.M. [etal.]Mechanism, assessment and management of pain in chronic pancreatitis: Recommendations of a multidisciplinary study group. Pancreatology. 2016; 16(1): 83-94. doi: 10.1016/j.pan.2015.10.015.

3. Ito T., Ishiguro H., Ohara H. et al. Evidence-based clinical practice guidelines for chronic pancreatitis 2015. Journal of Gastroenterology. 2016; 51(2):85-92. doi: 10.1007/s00535-015-1149-x.

4. Majumder S., Chari S.T. Chronic pancreatitis. Lancet. 2016 May 7;387(10031):1957-66. doi: 10.1016/S0140-6736(16)00097-0.

5. Kanikovskiy 0.E., Pavlyk I.V., Oliinyk I.V. The severity of pancreatic fibrosis - independent factor for choosing of type of surgical treatment in patients with chronic pancreatitis. Pancreatology. 2018;18 (4):167. (doi.org/10.1016/j.pan.2018.05.452)
6. Kanikosky 0.E., Pavlyk I.V., AndrosovS.I. et al. Definition of local resection margins in patients with chronic pancreatitis. 47 World Congress of Surgery 2017. Abstract book. 2017, p.302.

7. David C. Whitcomb, Luca Frulloni, Pramod Garg, et al. Chronic pancreatitis: An international draft consensus proposal for a new mechanistic definition. Pancreatology. 2016:16(2):218-224. Published online: doi: 10.1016/j.pan.2016.02.001

8. Bellin MD, Beilman GJ. Surgical trials for chronic pancreatitis. Lancet. 2017;390(10099):1007-1008. DOI: 10.1016/50140-6736(17)32338-3

9. Issa Y, van Santvoort HC, van Dieren S, Besselink MG, Boermeester MA, Ahmed Ali U. Diagnosing Chronic Pancreatitis: Comparison and Evaluation of Different Diagnostic Tools. - Pancreas. 2017;46(9):11581164. doi: 10.1097/MPA.0000000000000903.

\section{ORCID and contributionship:}

Oleh E. Kanikovskiy - 0000-0002-9302-8760 ${ }^{A, F}$

Ihor V. Pavlyk - 0000-0003-2018-273X $X^{A, B, D, E}$

Iryna V. Oliinyk - 0000-0001-6034-3580 ${ }^{C, E}$

Vasyl V. Mosondz-0000-0003-2821-7709 ${ }^{B, E}$

\section{Conflicts of interest:}

Authors declare no conflict of interest.

\section{CORRESPONDING AUTHOR \\ Ihor V. Pavlyk}

Department of surgery of medical faculty 2

National Pirogov memorial Medical University

56, Pyrogov str., 21031, Vinnytsia, Ukraine

tel: +380674194682

e-mail: ipavlyk@gmail.com

Received: 05.05.2019

Accepted: 25.11.2019

A - Work concept and design, B - Data collection and analysis, C - Responsibility for statistical analysis, D-Writing the article, $\mathbf{E}$-Critical review, $\mathbf{F}$ - Final approval of the article 